\title{
Don't let metonymy be misunderstood: An answer to Croft
}

\author{
YVES PEIRSMAN and DIRK GEERAERTS*
}

Misunderstandings are common, even among semanticists. Indeed, after having read William Croft's answer to our article, we believe that most of his criticisms arise from a misunderstanding of our intentions, and more broadly, of the type of model that we have developed. In this answer to Croft, we will first argue that our prototype model, as any prototype model in general, does not claim to put forward any necessary or sufficient attributes for the concept that it describes. Second, we will show that our use of contiguity is less controversial and less problematic than Croft claims. Third, we will counter Croft's criticism that our model is undermined by its own use of domains. Fourth, we will wrap up with some final remarks on Croft's defense of domain highlighting.

\section{On prototypicality}

Croft argues that "contiguity is neither a necessary nor a sufficient condition for metonymy", presenting a number of examples that fit into our model but that apparently constitute "ungrammatical" metonymies. There are at least three reasons why this line of reasoning is fundamentally misguided. First, it misreads the impact of a prototype-based analysis. Second, it is empirically incorrect. And third, it is based on a category mistake.

First, Croft's reaction is inspired by his search for a set of necessary and sufficient attributes that describe the ever-elusive phenomenon we call metonymy. The title of our article, in contrast, is Metonymy as a prototypical category. And the first characteristic of a prototypical category is, in Geeraerts' (1994: 3385) words, that it "cannot be defined by means of a single set of criterial (necessary and sufficient) conditions". Our point should be clear: never have we claimed to put forward a necessary and/or sufficient condition for metonymy. In fact, it is this type of definition that we react against: "This new stress on contiguity rather than on domains or domain matrices does not require us to use a unitary definition of 
contiguity. After all, the concept of contiguity is no less vague than that of domain or domain matrix" (p. 273). What we therefore suggest is to look at contiguity from a prototypical point of view. We thus put forward a prototypical core and a number of possible extensions from that core. The resulting model covers a wide variety of relationships that motivate specific metonymical patterns. Most of these relationships have been called "contiguous" (and thus, "metonymical") in the past, but they can certainly not be described by means of necessary and/or sufficient attributes.

Second, Croft argues that "[a]n analysis of a phenomenon such as metonymy must attempt to explain why some things are NOT acceptable metonymies as well as why other things are acceptable metonymies". However, his examples of "ungrammatical metonymies" are too restrictively chosen to make the point: their cross-linguistic range is too narrow, and they underestimate the impact of contextual flexibility on the acceptability of metonymies. To illustrate the cross-linguistic point, let us note that some of Croft's b-examples may be "ungrammatical metonymies" in English, but are not impossible in other languages. For instance, even though *fifty cardboards is disallowed in English, its Dutch equivalent vijftig kartons is perfectly acceptable (10b).

With regard to the contextual effects, observe that some of Croft's "ungrammatical" examples sound perfectly natural when they occur in an appropriate context. If you are throwing a big dinner party with hundreds of guests, for instance, you might very well say that the larger dining room is already eating dessert, while the smaller is still at the main course (5b). Such contextual metonymies are frequently discussed in the literature (cf. the well-known ham sandwich example), and we therefore believe that an explanatory model of metonymy should not restrict itself to the more conventional instances. After all, the difference can be seen as one of degree: one reason why a metonymy may become conventional is its applicability in many contexts. Our model abstracts away from this difference and tries to explain metonymy as a cross-linguistic and cross-contextual phenomenon.

Third, there are reasons of principle why our model is not undermined when some of the instances that it allows in theory do not occur in practice. Compare this with the biological category of "birds", which is generally accepted to have a prototypical structure. Prototypical attributes of a bird are, for instance, that it has wings and a beak, can fly, lays eggs, etc. Note that the existence of these attributes does not imply that all animals that they allow for in theory also exist in reality. And yet, their validity remains unchallenged: if we came across a new exemplar with these attributes, we would certainly call it a bird (assuming, for the sake of the argument, that there are no compelling reasons to range it with the mammals 
or the insects, for instance). Similarly, we would claim that, if Croft's example $1 \mathrm{~b}$ would actually occur, a linguist working in the tradition of lexical semantics would call it a metonymy, because it is motivated by contiguity. Whether or not the actual example occurs is a different matter, and does not undermine the description of the category. Croft thus makes a basic category mistake: the existence of contiguity as a motivation for something to be called a metonymy need not imply that this something actually exists. There is, in other words, a difference to be maintained between possibility and actuation, in the same way in which, for instance, a definition of diphthongization (whatever form the definition takes) does not specify which diphthongization processes will actually occur.

Clearly, putting matters in this way defines an additional research programme: what are the contextual factors that make the actuation of a possible metonymy more probable than the use of another mechanism of naming? And in addition, what are the factors that contribute to its conventionalization? Except for the fact that we would like to be careful with the difficult terminological pair semantic/pragmatic, we might call these "pragmatic" as opposed to "semantic" factors, and surely, it would be natural for Cognitive Linguistics to pay attention to such pragmatic factors. Kövecses and Radden (1998), for instance, present an overview of cognitive as well as communicative principles that influence the choice of the preferred metonymical vehicle. However, these matters are not what we focus on in our prototype-theoretical analysis.

\section{On contiguity}

Even though we do not present contiguity as a necessary and sufficient definition of metonymy, we still use it as the starting-point of the analysis. Two of Croft's points have to be addressed here: what evidence is there for choosing a certain type of contiguity as the core of the prototypical category, and why use "contiguity" and not "association"?

First, prototypical models come in different forms. Some are psychological, others are logical. Ours is the latter rather than the former: our article "presents a rational reconstruction of the relationships between the metonymical patterns in the inventory. It thus addresses the developments in the use of the term metonymy in the linguistic literature" (endnote 5). In other words, we have looked at the range of patterns that linguists have called "metonymical" in the past, and we have investigated how these patterns can be related to each other. Obviously, this would be a questionable approach for the development of a psychological model for the language user at large, which would need to rely on the results of carefully constructed psycholinguistic experiments instead. 
Psycholinguistic validity of that type, however, was never our aim. Therefore Croft's criticism that "no evidence is given that would indicate that some of P \& G's types of contiguity are more prototypical than others, in the form of graded acceptability judgements, order of mention, frequency of occurrence or of mention, etc." is simply misguided. The gradedness of our model resides in the tradition of diachronic semanticssome metonymical types are more often mentioned than others-and our paper is (as we make clear in no uncertain terms at the very beginning) an attempt to analyze the picture of metonymy as it emerges from this tradition.

The prototype category whose structure we analyze exists primarily in the mind of linguists. Whether it exists - consciously or subconsciouslyin the mind of language users is another matter, but at least, the fact that the linguists' category can be analyzed as a natural and coherent category suggests that it may indeed reflect a natural and coherent cognitive phenomenon.

Second, then, in our search for this coherence, contiguity presented itself as a valid starting point. This should not come as a surprise: since the very beginnings of the study of language, contiguity has been named as the primary motivation for metonymy. Strangely, this is questioned by Croft: "contiguity is an inferior description of metonymy in comparison to association, the traditional definition of metonymic semantic relations, since contiguity unlike association implies a notion of spatial nearness". We see two problems with this statement. First, if we look at some traditional definitions of metonymy, contiguity as well as association are mentioned frequently. Second, in cognitive linguistics, contiguity does not necessarily involve spatial nearness.

Let us go back a few thousand years, to the very beginnings of the rhetorical study of metonymy. The first attested definition of metonymy, in the anonymous work Rhetorica ad Herennium, describes it in terms of "near and close things". In the rhetorical tradition at least, this is synonymous for contiguity, as Panther and Thornburg (forthc.: 2) note in their forthcoming contribution to the Handbook of Cognitive Linguistics: "[t]his ancient characterization already points to the notions of contiguity and association that have ever since been criterial in distinguishing metonymy from metaphor".

Indeed, contiguity crops up time and again as the motivating factor for metonymy. Paul (1970: 83) refers to the metonymical target as "etwas, was mit dem usuellen Bedeutungsinhalt nach allgemeiner Erfahrung räumlich oder zeitlich oder kausal verknüpft ist [something which is in general experience spatially, temporally or causally linked with the traditional meaning]", Nyrop (1913: 188) defines metonymy as a "passage 
d'une représentation à une autre dont le contenu est avec la représentation donnée dans un rapport de contiguité [a transition from one representation to another whose content is in a contiguity relation with the first]", and Waag speaks of "einen thatsächlichen Zusammenhang, [...] eine erfahrungsgemässe reale Abhängigkeit des Raumes, der Zeit oder der Kasualität. [an actual connection,... an experiential dependence of space, time or cause]." Ullmann (1967: 232), finally, states that "[t]ransfers by contiguity between the senses are very common occurrences and have received extensive attention in the literature on semantics and rhetoric, where they correspond to the ancient categories of metonymy and synecdoche". All these definitions clearly demonstrate that Croft is simply incorrect in claiming that association, and not contiguity, has been the traditional definition of metonymy. Both have been present in the literature for a very long time.

Originally, contiguity was seen as spatial nearness, a perspective that Croft seems to take as well. In contrast, we are more in favour of the cognitive-linguistic view of contiguity, which we describe in the introduction of our article. In Dirven's (2002: 90-91) words, this view holds that "contiguity cannot in all cases be based on a form of objective or 'natural' contiguity. This has the implication that contiguity must be taken to mean 'conceptual contiguity' and that we can also have contiguity in those cases where we just 'see' contiguity between domains". Spatial nearness may be the prototypical core of contiguity, it does not exhaust this phenomenon, as Croft wishes to imply. In fact, a prototype structure with spatial nearness as the core (but not the delimiting case) of the concept is clearly present in the quotes we presented above: nearly all of them refer to other relations in addition to spatial ones, but at the same time, they all mention spatial relations as the initial (and probably most conspicuous) case.

\section{On domains}

Given the supposed disadvantages of contiguity, Croft defends his domain highlighting approach to metonymy. Our discussion of this topic, he argues, is too short to allow for a fair evaluation, and what is more, the credibility of our model is undermined by its own use of domains. Again, we disagree.

Why did we spend so little time describing the domain definition of metonymy? There is a simple answer to that question: our article was not meant as a detailed critique of that definition. Rather, it investigated a possible alternative that had not yet been looked into. There were obvious reasons for doing so: quite a number of linguists have expressed 
their dissatisfaction with the domain approach. Among them are Feyaerts and Riemer, but also Croft and Cruse, despite Croft's claim to the opposite. On page 216 of their book, we read that "[m]etonymy is distinguished [from metaphor] by the fact that (i) A and B are associated in some domain or domain matrix [...]". To this, Croft and Cruse add the following footnote: "[w]hile identity of domain does seem to be a factor, we agree with Feyaerts (2000) and Riemer (2001) that on its own it remains an unreliable criterion in the absence of independent means of delimiting domains". This seems to us an unambiguous criticism of the domain definition. We understand that Croft may not have read all the details of our text with the same attention (cp. above), but we think we may expect that he quotes his own texts correctly.

While it is true that our model still incorporates domains, this does not mean that it uses them in the same way as the domain highlighting definition of metonymy. We are not concerned with profiles or bases, but rather, with the domains in which contiguity is possible, and the mappings between them - in much the same way, in fact, as mappings between domains are used in cognitive-linguistic metaphor analyses. By the way, we wanted to avoid any confusion with the earlier use of domains in the definition of metonymy by calling the domains we invoke "conceptual realms", but it looks like this measure of caution was not sufficiently strong.

More importantly, we do not claim that a definition of metonymy in terms of domains has to be avoided at all costs: we explicitly state that our prototype-based analysis is not incompatible with a schematic unitary definition (p. 311), which could take the form of an analysis in terms of domain highlighting. However, we do believe that a description of metonymy in terms of domain highlighting suffers from some major shortcomings. We did not mean to pursue this matter in great detail in the original article, but since Croft challenges us, we will now happily add a few of our reservations with respect to domain highlighting.

\section{On domain highlighting}

While a detailed analysis and critique of the domain highlighting hypothesis would doubtlessly lead to another long article, we will just mention a number of objections that should certainly be included in such a debate. First, like our model, Croft's definition is not able to rule out the "ungrammatical" metonymies he presents. Second, Croft's approach to metonymy is unnecessarily restrictive and rests on circular reasoning. Third, we still believe that it explains many examples after the fact only. And fourth, it does not lead to a classification of types of 
metonymy that corresponds to what generations of linguists have found to be useful.

In section 1, we already hinted that many of Croft's "ungrammatical metonymies" are as problematic for his definition as for ours. Croft claims that *I painted the fridge (for "kitchen", 6b) is ungrammatical because it does not display a shift in domain highlighting. Let us compare it to our example sich einen eignen Herd gründen (to build one's own hearth for "to build one's own house"). This example is not contested by Croft, and so here, apparently, domain highlighting is possible. But isn't the relationship between a hearth and a house very similar to that between a fridge and a kitchen? And if so, why is domain highlighting allowed in the latter, but not in the former? Similarly, why can a container refer to the drink it contains in a sentence like I drank a glass too many (3a), but not in The glass is red (3b)? Croft argues this is because the former displays domain highlighting, whereas the latter does not. But this reasoning is circular: it does not answer the question why domain highlighting sometimes occurs, and sometimes not. It is true that pragmatic questions like these cannot be answered by our model (as we discussed above), but on this point Croft's approach fails just as well. Remember that he thinks the analysis should indeed rule out such ungrammatical cases, while we believe that the analysis should rather delimit the possible cases of metonymy. The actuation and the acceptability of actual cases could depend on additional "pragmatic" factors.

Second, Croft appears to have problems with our treatment of too wide a variety of metonymical patterns. He argues that examples like ein Mädchen zum Altar führen, or Can you pass the salt actually represent another cognitive phenomenon. Why? Because they do "not involve the Conceptual Unity of Domain" (which typically applies within the clause) and hence, they do not "involve a shift in domain highlighting". This is again a circular argument. Croft constructs a definition of metonymy, and then calls examples like those above non-metonymical because they do not fit into his definition. As we have already discussed, our perspective is crucially different: we do not intend to reform the definition of metonymy, but we merely wish to analyze the existing usage of the term. Since all of the examples in our inventory have been called metonymical in the literature, we assume there must be something that relates all of them, and not just some.

The main objection we raised with regard to a domain analysis of metonymy was that, with contextual metonymies in particular, it is often possible after the fact only. We illustrated this with the Italian example moneta. Croft disagrees on this point, and claims that "any analysis of social 
domains would distinguish religion from economy". This is precisely our reason for distrusting the domain approach: it seems very unlikely that anyone would include the religious concept of "goddess" and the economic concept of "coins" within the same domain matrix, unless they already knew that a metonymical shift between them had occurred or was going to occur. Similarly, take the well-known ham sandwich metonymy, which involves a shift from the food domain to the restaurant domain. Does the meaning of "ham sandwich" really presuppose the restaurant domain in its base, as Langacker's definitions of domains and domain matrices would require? Given the encyclopedic nature of meaning in Cognitive Semantics, the inclusion of the restaurant domain in the domain matrix of ham sandwiches is certainly not impossible, but we do not expect it to happen automatically. It's like Schrödinger's cat, really: whether there is a domain to be highlighted becomes clear only after the light has already been switched on.

With these problems in mind, is easy to see why the exploration of an alternative approach to metonymy is justified, to say the least. What is more, we insist on repeating that the enthusiastic supporters of the domain approach need not despair: our model and theirs are not necessarily incompatible. Even they will have to admit that the domain highlighting definition remains silent on the typical relationships that exist between the original domain and the highlighted domain. Saying that the former presupposes the latter does not shed any light on the concrete motivations there may be for including two domains within the same matrix. It surely does not lead to a classification of these possible motivations. Our prototypical model of contiguity and metonymy suggests such a classification and shows how the possible links between the original domain and the highlighted domain can be related to one another. This perspective should appeal even to the most radical proponents of the domain highlighting approach.

But of course, we believe our model does more. It reacts against the ongoing search for a definition that describes metonymy in terms of necessary and/or sufficient attributes. It argues against the resulting, reductionist tendency to restrict metonymy to a handful of patterns and to argue that the many linguists who did otherwise simply were wrong. It questions the domain definition of metonymy, which all too often reflects an interpretation of the semantic shift after the fact. And it manages to explain a wide variety of patterns that have previously been called metonymical on the basis of a prototype-based analysis of contiguity. That should be enough to take it seriously, should it not? 


\section{Note}

* The authors are affiliated with the University of Leuven, Research Group Quantitative Lexicology and Variational Linguistics, Blijde-Inkomststraat 21, 3000 Leuven, Belgium. Authors' e-mail addresses:〈yves.peirsman@arts.kuleuven.be〉,〈dirk.geeraerts@ arts.kuleuven.be $>$.

\section{References}

Croft, W. and D. A. Cruse

2002 Cognitive Linguistics. Cambridge: Cambridge University Press.

Dirven, R.

2002 Metonymy and metaphor: Different mental strategies of conceptualisation. In Dirven, R. and R. Pörings (eds.), Metaphor and Metonymy in Comparison and Contrast. (Cognitive Linguistics Research 20.) Berlin/New York: Mouton de Gruyter, 75-111.

Feyaerts, K.

2000 Refining the inheritance hypothesis: Interaction between metaphoric and metonymic hierarchies. In Barcelona, A. (ed.), Metaphor and Metonymy at the Crossroads: A Cognitive Perspective. Berlin/New York: Mouton de Gruyter, 59-78.

Geeraerts, D.

1994 Prototype semantics. In Asher, R. E. (ed.), The Encyclopedia of Language and Linguistics. Oxford: Pergamon Press, 3384-3386.

Kövecses, Z. and G. Radden

1998 Metonymy: Developing a cognitive linguistic view. Cognitive Linguistics 9, $37-77$.

Nyrop, K.

1913 Grammaire historique de la langue française. Copenhagen: Gyldendalske Boghandel, Nordisk Forlag.

Panther, K.-U. and L. Thornburg

In press Metonymy. In Geeraerts, D. and H. Cuyckens (eds.), Handbook of Cognitive Linguistics. Oxford/New York: Oxford University Press.

Paul, H.

$1970 \quad$ Prinzipien der Sprachgeschichte. Tübingen, Max Niemeyer Verlag.

Riemer, N.

2001 Remetonymizing metaphor: Hypercategories in semantic extension. Cognitive Linguistics 12, 379-401.

Ullmann, S.

1967 The Principles of Semantics. (Glasgow University Publications 84.) Oxford: Blackwell. 
Authenticated | dirk.geeraerts@arts.kuleuven.be author's copy Download Date | 4/13/12 7:34 PM 